\title{
Impact of Plasma Glucose Level at the Time of Fluorodeoxyglucose Administration on the Accuracy of FDG-PET/CTin the Diagnosis of Pancreatic Lesions
}

\author{
Alireza Hamidian Jahromi ${ }^{1}$; Mohammad Kazem Fallahzadeh ${ }^{2}$; Amol Takalkar ${ }^{3}$; Jean Sheng ${ }^{1}$; \\ Gazi Zibari ${ }^{2}$; Hosein Shokouh Amiri ${ }^{2, *}$ \\ ${ }_{2}^{1}$ Department of Surgery, Louisiana State University, Shreveport, Louisiana, USA \\ ${ }^{2}$ John C. McDonald Regional Transplant Center, Willis Knighton Health System, Shreveport, Louisiana, USA \\ ${ }^{3}$ Department of Nuclear Medicine, Louisiana State University, Shreveport, Louisiana, USA \\ ${ }^{*}$ Corresponding author: Hosein Shokouh Amiri, John C. McDonald Regional Transplant Center, Willis Knighton Health System, Shreveport, Louisiana, USA. Tel: +1-3182128932, Fax: \\ +1-3182128356, E-mail: hshokouh@wkhs.com
}

Received: December 5, 2013; Revised: March 16, 2014; Accepted: April 12, 2014

\begin{abstract}
Background: High fasting plasma glucose (FPG) levels before fluorodeoxyglucose (FDG) administration for positron emission tomography/computed tomography (PET/CT) might affect the accuracy of 18-fluoro-deoxy-glucose-positron emission tomographycomputed tomography (FDG-PET/CT) in diagnosis of pancreatic lesions. Current guidelines require FPG levels of $<200 \mathrm{mg} / \mathrm{dL}$ before FDG administration; however, the literature on the effect of FPG levels of $<200 \mathrm{mg} / \mathrm{dL}$ on the accuracy of FDG-PET/CT is scarce.

Objectives: The aim of this study was to evaluate the effect of FPG levels of $<200 \mathrm{mg} / \mathrm{dL}$ on the accuracy of FDGPET/CT in diagnosis of pancreatic lesions.

Patients and Methods: In this retrospective study, 161 patients who had FDG-PET/CT for initial diagnosis of pancreatic lesions were included. Fasting plasma glucose levels before FDG administration were recorded. Accuracy of FDG-PET/CT in diagnosis of pancreatic lesions was compared between patients who were normoglycemic (FPG $<126 \mathrm{mg} / \mathrm{dL}$ ) and hyperglycemic (126 $\leq$ FPG $<200 \mathrm{mg} / \mathrm{dL}$ ).

Results: Thirty-four patients were hyperglycemic and 127 normoglycemic. Sensitivity, specificity, positive predictive value and negative predictive value of FDG-PET/CT were $90 \%, 88 \%, 87 \%$ and $91 \%$ in normoglycemic and $82 \%, 92 \%, 95 \%$ and $73 \%$ in hyperglycemic patients, respectively. Overall, the accuracy was higher in normoglycemic than hyperglycemic patients (89\% vs. $85 \%$ ).

Conclusions: Accuracy of FDG-PET/CT for primary diagnosis of pancreatic lesions is higher in patients with FPG levels $<126 \mathrm{mg} / \mathrm{dL}$ than in patients with FPG levels between 126 and $200 \mathrm{mg} / \mathrm{dL}$.
\end{abstract}

Keywords:Cancer; Diagnosis; Glucose; Pancreas; Positron Emission Tomography

\section{Background}

Following a successful introduction phase, 18-fluoro-deoxy-glucose-positron emission tomography-computed tomography (FDG-PET/CT) is now emerging as a useful imaging tool in the diagnosis and management of the patients with pancreatic lesions $(1,2)$. Although previous studies have reported high sensitivity and specificity for FDG-PET/CT in the differentiation of benign and malignant pancreatic pathologies, there is no current consensus on the role of FDG-PET/CT in the initial diagnostic work up (and subsequently planning initial treatment strategy) for pancreatic lesions (3). The 18-fluoro-deoxyglucose (FDG) is a radiolabeled glucose analog used in FDG-PET/CT, which competes with plasma glucose for uptake in different tissues. Increased expression of glucose transporter (GLUT-1) in pancreatic tumor cells compared with normal/inflamed non-cancerous pancreatic cells (pancreatitis) and the pattern of the glucose uptake in the pancreas (focal high uptake in pancreatic cancer as opposed to diffuse high uptake in pancreatitis) are the main pathophysiological mechanisms used in FDG-PET/ CT for differentiation of malignant and benign pancreatic pathologies. Previous studies have suggested that high fasting plasma glucose (FPG) levels just prior to FDG administration for FDG-PET/CT might affect the accuracy of this modality in response predictions in rectal cancers as well as efficacy of this modality in diagnosis of cervical cancer $(4,5)$. Lindholm et al. showed that the standardized uptake values (SUV) and Ki and consequently the quality of the positron emission tomography (PET) image become markedly poorer after oral glucose loading to the patients (6). Although Diederichs and colleagues, acknowledged the improved diagnostic yield of PET-only scan for differentiation of malignant and benign pancreatic masses of patients with FPG values less than $130 \mathrm{mg} /$ $\mathrm{dL}$, this concept has not been recently evaluated for the diagnostic accuracy of FDG-PET/CT scans in detection of pancreatic pathologies (7). It is well established that high plasma glucose levels at the time of FDG administration

Copyright (C) 2014, Research Institute For Endocrine Sciences and Iran Endocrine Society; Published by Kowsar. This is an open-access article distributed under the terms of the Creative Commons Attribution-NonCommercial 4.0 International License (http://creativecommons.org/licenses/by-nc/4.0/) which permits copy and redistribute the material just in noncommercial usages, provided the original work is properly cited. 
affects FDG bio-distribution. The current guidelines for FDG-PET/CT imaging requires the patient to fast for at least six hours prior to FDG-PET/CT imaging and the fasting plasma glucose (FPG) levels to be $\leq 200 \mathrm{mg} / \mathrm{dL}$ at the time of 18-FDG administration (8). We are currently lacking information on accuracy of FDG-PET/CT in lower plasma glucose (PG) levels ( $\leq 200 \mathrm{mg} / \mathrm{dL}$ ); i.e. the diagnostic value of FDG-PET/CT for detection and differentiation of pancreatic pathologies in normoglycemic patients with FPG $<126 \mathrm{mg} / \mathrm{dL}$ versus patients with FPG $\leq 200 \mathrm{mg} / \mathrm{dL}$ who are not normoglycemic, i.e. FPG 126-200 mg/dL labeled as hyperglycemic for this study.

\section{Objectives}

The aim of our study was to evaluate the impact of fasting plasma glucose level (i.e. normoglycemic versus hyperglycemic) at the time of FDG administration on the accuracy of FDG-PET/CT in the evaluation of suspicious pancreatic lesions.

\section{Patients and Methods}

In this retrospective Institutional Review Board (IRB)approved study, charts of all patients who had FDG-PET| CT from 2007 to 2011 at our center for evaluation of pancreatic lesion were reviewed. Among these candidates, patients who had FDG-PET/CT for initial diagnostic workup of pancreatic lesions (i.e. evaluation of a pancreatic lesion without confirmed tissue diagnosis) were included. Demographics, final pathological diagnosis and FPG levels at the time of FDG administration were recorded. The FPG was confirmed to be less than $200 \mathrm{mg} / \mathrm{dL}$ before proceeding with the FDG-PET/CT scan. A board certified nuclear medicine physician blinded to the final diagnosis and FPG levels evaluated all FDG-PET/CT images. FDG-PET/CT images were evaluated subjectively (visual analysis) as well as objectively (by obtaining SUV). Based on visual analysis, lesions that had focal intense FDG uptake were deemed suspicious for malignant etiology and lesions with no FDG uptake or with mild and diffuse uptake over a larger area of the pancreas with heterogeneity and adjacent fat/ soft tissue stranding were deemed more likely to be benign. Subsequently, the maximum and average standardized uptake value (SUV-max and SUV-average) of these abnormal foci was also obtained using volume of interest methodology inbuilt in the workstation utilized to review these scans (GE ADW 4.3, GE Medical, Milwaukee, WI).

\subsection{Statistical Analysis}

Descriptive statistics were used to summarize the data. Statistical analysis was performed using the SPSS software version 15 (SPSS Inc., Chicago, IL). For all lesions that were suspicious based on clinical opinion (that considered FDG PET/CT findings), final diagnoses were confirmed by cytology or tissue diagnosis. For lesions that were favored to be benign or inflammatory based on clinical opinion, final diagnoses were determined by tissue diagnosis and/ or clinical course. The diagnoses initially done by the nuclear medicine physician were compared with the final diagnoses. Lesions with final diagnosis of pancreatic adenocarcinoma or neuroendocrine tumor were designated as true positive (TP) if they were correctly diagnosed by the nuclear medicine physician and were designated as false negative (FN) if they were incorrectly diagnosed as benign or inflammatory lesions by the nuclear medicine physician. Similarly, lesions with final diagnosis of benign or inflammatory were designated as true negative (TN) if they were correctly diagnosed by the nuclear medicine physician and were designated as false positive (FP) if they were incorrectly diagnosed as pancreatic adenocarcinoma or neuroendocrine tumor by the nuclear medicine physician. The sensitivity of FDG-PET/CT in diagnosis of malignant versus benign pancreatic lesions was calculated by dividing the number of TP cases by the number of all patients with pancreatic adenocarcinoma and neuroendocrine tumor. Specificity was calculated through dividing the number of TN cases by the number of all patients with benign and inflammatory lesions. Positive predictive value (PPV) was calculated through dividing the number of TP cases by the number of all cases who were diagnosed as pancreatic adenocarcinoma or neuroendocrine tumor on FDG-PET/CT. Negative predictive value (NPV) was calculated by dividing the number of TN cases to the number of all cases who were diagnosed as benign or inflammatory lesions on FDG-PET/CT. The total accuracy of FDG-PET/ CT was calculated through dividing the number of all TN and TP cases by the number of all patients enrolled in this study. Moreover, sensitivity, specificity, and accuracy of FDG-PET/CT in diagnosis of malignant versus benign pancreatic lesions were calculated in a similar manner for patients who were normoglycemic (FPG $<126 \mathrm{mg} / \mathrm{dL}$ ) and also for patients who were hyperglycemic (FPG 126-200 $\mathrm{mg} / \mathrm{dL}$ ) at the time of FDG administration.

\section{Results}

From 2007 to 2011, 161 patients who had FDG-PET/CT for initial diagnosis of pancreatic lesions were included in the study. Demographic and pathological characteristics of the patients are shown in Table 1. Seventy-eight (48.4\%) patients had pancreatic adenocarcinoma (69 (88.5\%) were true positives (TP) and nine (11.5\%) false negatives (FN) by FDG-PET/CT), five (3.1\%) had neuroendocrine tumors (four (80\%) TP and one (20\%) FN by FDG-PET/CT), and $78(48.4 \%)$ had other benign or inflammatory pancreatic pathologies (69 (88.5\%) true negative (TN) and nine (11.5\%) false positive (FP) by FDG-PET/CT).

Sensitivity, specificity, PPV and NPV of FDG-PET/CT in the diagnosis of pancreatic lesions were $88 \%, 88 \%, 89 \%$ and $87 \%$, respectively. The overall accuracy of FDG-PET/CT in the diagnosis of pancreatic lesions was $88 \%$. The PG levels of all patients were below $200 \mathrm{mg} / \mathrm{dL}$ at the time of FDG administration. One hundred and twenty-seven (79\%) 
Table 1. Demographic and Pathological Characteristics of Normoglycemic (FPG $<126 \mathrm{mg} / \mathrm{dL})$, Hyperglycemic $(126 \leq$ FPG $<200 \mathrm{mg} /$ dL) and All Patients ${ }^{\mathrm{a}}$

\begin{tabular}{lccc}
\hline $\begin{array}{l}\text { Demographic and Pathology } \\
\text { Characteristics }\end{array}$ & Normoglycemic Patients $(\mathbf{n}=\mathbf{1 2 7})$ & Hyperglycemic Patients $(\mathbf{n}=\mathbf{3 4})$ & All Patients $(\mathbf{n}=\mathbf{1 6 1})$ \\
\hline Age, $\mathbf{y}$ & $60.2 \pm 13.6$ & $62.5 \pm 13.9$ & $60.7 \pm 13.6$ \\
\hline $\begin{array}{l}\text { Male } \\
\text { Pathology }\end{array}$ & $72(56.7)$ & $19(55.9)$ & $91(56.5)$ \\
\hline $\begin{array}{l}\text { Pancreatic adenocarcinoma } \\
\text { Neuroendocrine tumors }\end{array}$ & $56(44.1)$ & $22(64.7)$ & $78(48.4)$ \\
\hline $\begin{array}{l}\text { Other benign/inflammatory } \\
\text { lesions }\end{array}$ & $5(3.9)$ & $0(0)$ & $5(3.1)$ \\
\hline
\end{tabular}

${ }^{\mathrm{a}}$ Data are presented as Mean \pm SD or No. (\%).

patients were normoglycemic and 34 (21\%) were hyperglycemic. The 18F-FDG-PET/CT in normoglycemic patients had sensitivity, specificity, PPV and NPV of 90\%, 88\%, $87 \%$ and $91 \%$, respectively. The FDG-PET/CT in hyperglycemic patients had sensitivity, specificity, PPV and NPV values of $82 \%, 92 \%, 95 \%$ and $73 \%$, respectively. Overall, the accuracy of FDG-PET/CT was higher in normoglycemic patients compared to the hyperglycemics ( $89 \%$ vs. $85 \%$ ) but the specificity was higher in hyperglycemic patients compared to normoglycemics ( $92 \%$ vs. $88 \%$ ).

\section{Discussion}

In our study, FDG-PET/CT had a higher sensitivity but a lower specificity for diagnosis of pancreatic lesions in normoglycemic patients compared with hyperglycemics. Furthermore, FDG uptake in various tissues including tumor cells is highly susceptible to the internal hormonal milieu, especially in relation to plasma insulin levels and plasma glucose levels. Eighteen-fluoro-deoxy-glucose is a glucose analogue and follows the pathways of glucose metabolism to a certain extent. Similar to regular glucose, FDG is transported intracellularly via GLUT-1 through GLUT-4 receptors $(9,10)$. Once inside the cells, again similar to glucose, FDG is acted upon by the enzyme hexokinase and is converted to FDG-6-phosphate (9-11). However, subsequent processing of FDG-6-pohosphate does not proceed along the lines of glucose-6-phosphate as the downstream enzymes (like glucose-6-phosphatase) are more specific and have less affinity for FDG-6-phosphate (11). Thus, FDG remains metabolically trapped inside the cells. Since cancer cells prefer anaerobic glycolysis and have up-regulation of GLUT receptors, there is more accumulation of FDG in cancer cells as compared to normal cells; hence, these cancer cells show intense FDG uptake on FDG-PET/CT imaging. However, uptake of FDG by various tissues in the body including cancer cells can be affected by several factors. Insulin decreases plasma glucose levels by driving glucose into muscles. Therefore, if the plasma insulin levels are high at the time of FDG administration (either in response to recent intake of food by the patient or due to administration of exogenous insulin for controlling blood glucose levels), insulin will also drive FDG (similar to glucose) into muscles and alter FDG bio-distribution with potentially less FDG remaining in the plasma to enter tumor cells. If there is high plasma glucose level (endogenous plasma glucose) at the time of FDG administration, there will be competition between FDG and endogenous glucose for the GLUT receptors and less potential for FDG to enter tumor cells. In the above scenarios, there is a potential for false negative scans as FDG uptake in various cells (including tumor cells) is affected. This is the underlying rational for requiring patients to fast for at least six hours prior to FDG-PET/CT imaging and to ensure that FPG is less than $200 \mathrm{mg} / \mathrm{dL}$ (without recent insulin administration) at the time of FDG administration. However, normal FPG is $<126 \mathrm{mg} / \mathrm{dL}$ and hence there is a theoretical possibility that even in patients with FPG $<200$ but higher than $126 \mathrm{mg} / \mathrm{dL}$ at the time of FDG administration (that is in compliance with the current guidelines), there may still be an inappropriately high level of competition between endogenous glucose and FDG. The decreased sensitivity and accuracy in hyperglycemic patients compared to normoglycemic patients in our study confirms and reinforces this mechanism. Interestingly, hyperglycemic patients in our study showed a higher specificity compared with normoglycemic patients. We postulate the following underlying reason for this finding. In hyperglycemic patients with ongoing competition between endogenous glucose and FDG, cells that show focal intense FDG uptake are more likely to be tumor cells with up-regulated GLUT-1 receptors. Benign or inflammatory cells without up-regulation of GLUT-1 receptors are unlikely to show focal intense FDG uptake in this setting, thereby diminishing the possibility of false positive scans and increasing specificity (7). The main limitation of our study was its small sample size. The findings of our study need to be further evaluated and confirmed by larger studies. If confirmed by future prospective studies with larger sample sizes, our findings could potentially have significant clinical implications in our daily practice when performing FDG-PET/CT imaging for diagnostic and follow-up evaluation of pancreatic lesions. Modifications of the FDG-PET/CT protocols to include tighter regulation of the pre-imaging FPG level and possible correction of the pre-imaging FDG to normogly- 
cemic levels (FPG levels $<126 \mathrm{mg} / \mathrm{dL}$ ) are examples of such implications. Nuclear medicine physicians/radiologists may also consider the pre-imaging FPG levels in their interpretations of FDG-PET/CT imaging. Normoglycemic pre-imaging FPG levels can result in higher sensitivity of FDG-PET/CT in detecting pancreatic lesions; on the other hand, hyperglycemic pre-imaging FPG levels can result in higher specificity of FDG-PET/CT in detecting pancreatic lesions.

In accordance with previous reports, our results show that FDG-PET/CT has high accuracy, sensitivity, specificity, PPV and NPV in the initial diagnosis and differentiation of malignant from benign pancreatic lesions. Overall, the accuracy of 18F-FDG-PET/CT was higher in normoglycemic patients compared with hyperglycemics; however, specificity was higher in hyperglycemic patients compared with normoglycemics.

\section{Acknowledgements}

The authors would like to thank Mr. John Cyrus who provided us editorial assistance.

\section{Author's Contributions}

1. Study concept and design: Alireza Hamidian Jahromi, Mohammad Kazem Fallahzadeh, Takalkar, Sheng, Zibari, Shokouh Amiri; 2. Acquisition of data: Alireza Hamidian Jahromi, Mohammad Kazem Fallahzadeh, Takalkar, Sheng; 3. Analysis and interpretation of data: Alireza Hamidian Jahromi, Mohammad Kazem Fallahzadeh, Takalkar, Sheng, Zibari, Shokouh Amiri; 4. Drafting of the manuscript: Alireza Hamidian Jahromi, Mohammad Kazem Fallahzadeh, Takalkar, Sheng, Zibari, Shokouh Amiri; 5. Critical revision of the manuscript for important intellectual content: Alireza Hamidian Jahromi, Mohammad Kazem Fallahzadeh, Takalkar, Sheng, Zibari, Shokouh Amiri; 6. Statistical analysis: Alireza Hamidian Jahromi,
Mohammad Kazem Fallahzadeh.

\section{References}

1. Grassetto G, Rubello D. Role of FDG-PET/CT in diagnosis, staging, response to treatment, and prognosis of pancreatic cancer. Am J Clin Oncol. 2011;34(2):111-4.

2. Asagi A, Ohta K, Nasu J, Tanada M, Nadano S, Nishimura R, et al. Utility of contrast-enhanced FDG-PET/CT in the clinical management of pancreatic cancer: impact on diagnosis, staging, evaluation of treatment response, and detection of recurrence. Pancreas. 2013;42(1):11-9.

3. De Gaetano AM, Rufini V, Castaldi P, Gatto AM, Filograna L, Giordano A, et al. Clinical applications of (18)F-FDG PET in the management of hepatobiliary and pancreatic tumors. Abdom Imaging. 2012;37(6):983-1003.

4. Janssen MH, Ollers MC, van Stiphout RG, Riedl RG, van den Bogaard J, Buijsen J, et al. Blood glucose level normalization and accurate timing improves the accuracy of PET-based treatment response predictions in rectal cancer. Radiother Oncol. 2010;95(2):203-8.

5. Chang YC, Yen TC, Ng KK, See LC, Lai CH, Chang TC, et al. Does diabetes mellitus influence the efficacy of FDG-PET in the diagnosis of cervical cancer? Eur J Nucl Med Mol Imaging. 2005;32(6):647-52.

6. Lindholm P, Minn H, Leskinen-Kallio S, Bergman J, Ruotsalainen $\mathrm{U}$, Joensuu $\mathrm{H}$. Influence of the blood glucose concentration on FDG uptake in cancer--a PET study. J Nucl Med. 1993;34(1):1-6.

7. Diederichs CG, Staib L, Vogel J, Glasbrenner B, Glatting G, Brambs $\mathrm{HJ}$, et al. Values and limitations of 18F-fluorodeoxyglucose-positron-emission tomography with preoperative evaluation of patients with pancreatic masses. Pancreas. 2000;20(2):109-16.

8. Caobelli F, Pizzocaro C, Paghera B, Guerra UP. Proposal for an optimized protocol for intravenous administration of insulin in diabetic patients undergoing (18)F-FDG PET/CT. Nucl Med Commun. 2013;34(3):271-5.

9. Ong LC, Jin Y, Song IC, Yu S, Zhang K, Chow PK. 2-[18F]-2-deoxy-D-glucose (FDG) uptake in human tumor cells is related to the expression of GLUT-1 and hexokinase II. Acta Radiol. 2008;49(10):1145-53.

10. Zhao S, Kuge Y, Tsukamoto E, Mochizuki T, Kato T, Hikosaka K, et al. Fluorodeoxyglucose uptake and glucose transporter expression in experimental inflammatory lesions and malignant tumours: effects of insulin and glucose loading. Nucl Med Commun. 2002;23(6):545-50.

11. Khan N, Islam MM, Mahmood S, Hossain GA, Chakraborty RK. $18 \mathrm{~F}-$ fluorodeoxyglucose uptake in tumor. Mymensingh Med $\mathrm{J}$. 2011;20(2):332-42. 